\title{
Effect of Synaid on cognitive functions and mood in elderly subjects with self-perceived loss of memory after COVID-19 infection
}

Marilisa Bove ${ }^{1}$, Federica Fogacci ${ }^{2}$, Silvia Quattrocchi ${ }^{1}$, Maddalena Veronesi ${ }^{1}$, Arrigo F.G. Cicero ${ }^{2}$

${ }^{1}$ IRCCS Azienda Ospedaliero-Universitaria di Bologna, Bologna, Italy ${ }^{2}$ Medical and Surgical Sciences Department, University of Bologna, Bologna, Italy

Submitted: 2 May 2021; Accepted: 22 August 2021

Online publication: 17 September 2021

Arch Med Sci 2021; 17 (6): 1797-1799

DOI: https://doi.org/10.5114/aoms/141502

Copyright $\odot 2021$ Termedia \& Banach

\section{Abstract}

Introduction: There is growing attention towards the possible long-term effect of COVID-19 on neuropsychiatric disorders.

Methods: We tested the effect of a combined nutraceutical (Synaid) with known nootropic effects in 40 elderly patients with self-perceived cognitive decline after SARS-CoV-2 infection, treated at home or in hospital.

Results: After 3 months of treatment, Synaid significantly improved functional status, MMSE and the main psychological disorders associated with mild cognitive impairment, both in patients previously cared for at home and in hospital because of SARS-CoV-2 infection.

Conclusions: Synaid was well tolerated and effective in improving cognitive function and psychosocial parameters after SARS-CoV-2 infection.

Key words: cognitive function, COVID-19, nutraceutical, dietary supplement.

Acute consequences of SARS-CoV-2 infection have gained great attention in the scientific literature, particularly regarding respiratory and cardiovascular effects [1]. Nonetheless, there has been noted increasing consideration towards the possible long-term effect of COVID-19 on neurological and psychiatric disorders, as well. Some reports have shown that cognitive impairments can also begin in patients recovered from COVID-19, which is likely to be linked to the underlying inflammatory processes [2].

In fact, severely affected COVID-19 patients experienced high levels of proinflammatory cytokines and acute respiratory dysfunction, and they often required assisted ventilation. Straightforward negative effects of the immune reaction, an acceleration or an aggravation of pre-existing cognitive deficits, or a new induction of a neurodegenerative disease could cause cognitive decline [3]. In particular, the hippocampus seems to be more vulnerable to coronavirus infections, which increase the probability of post-infection memory impairment and acceleration of neurodegenerative disorders associated with cognitive decline [4].

During the COVID-19 pandemic, Italy was one of the Western countries most severely hit [5]. Consequently, we expect a considerable number of long-term health consequences in the coming years.

In this pilot study, we aimed to demonstrate the positive effect of a combined nutraceutical with known nootropic effects [6] in patients

\author{
Corresponding author: \\ Prof. Arrigo F.G. Cicero \\ Medical and Surgical \\ Sciences Department \\ University of Bologna \\ Bologna, Italy \\ Phone: +39 3498558017 \\ E-mail: arrigo.cicero@unibo.it
}


with self-perceived cognitive decline after SARSCoV-2 infection.

Methods. For this purpose, we enrolled 40 elderly subjects (mean age: $68 \pm 3$ ) with an MMSE score at baseline between 20 and 27, recovered from COVID during the last 6 months, and with subjective cognitive decline after SARS-CoV-2 infection.

Exclusion criteria were:

1) Previous diagnosis of cognitive decline or dementia,

2) Confirmed diagnosis of dementia at the enrolment visit,

3) Personal history of cardiovascular diseases or diabetes,

4) Blood pressure or LDL cholesterol values out of target,

5) Any pharmacological treatment potentially affecting cognitive performance.

Patients could have experienced hospital care or home care.

All patients were treated with Synaid, a combined nutraceutical, containing Bacopa monnieri dry extract $320 \mathrm{mg}$ (bacosides $80 \mathrm{mg}$ ), L-theanine $100 \mathrm{mg}$, saffron $30 \mathrm{mg}$ (crocin $1.5 \mathrm{mg}$ ), vitamin $B_{6}$ $9.5 \mathrm{mg}$, vitamin D $25 \mu \mathrm{g}$, copper $2 \mathrm{mg}$, biotin $450 \mu \mathrm{g}$, folic acid $400 \mu \mathrm{g}$, and vitamin $\mathrm{B}_{12} 33 \mu \mathrm{g}$ (INPHA Duemila Srl, Milano, Italy), 1 capsule per day, for 12 weeks.

The following questionnaires were submitted at the baseline and at the end of the study: Mini-Mental State Examination (MMSE), Perceived Stress Questionnaire (PSQ) Index, and Self-Rating Depression Scale (SRDS).

Participants were tested during a morning visit in a peaceful room by neuro-psychologically trained research assistants who were not directly involved in the study. Cognitive testing was evaluated by MMSE, a widely used screening tool for cognitive impairment that covers 5 areas of cognitive function (orientation, attention, calculus, recall and language), with a score ranging from 0 to 30. MMSE was adjusted by age and education. The value of 27 of the MMSE score has been typically considered the threshold of normal cognitive function [7]. Then, the PSQ Index [8] and SRDS [9] were administered to the same subjects at the beginning and at the end of the study, as well. Finally, participants had to complete the Post-COVID-19 Functional Status (PCFS) scale, which has become a validated tool to measure functional status over time after COVID-19 [10].

Synaid tolerability was also tested at the end of the trial with a semi-quantitative scale.

Throughout the study, we instructed patients to take the first dose of the new product the day after the study product's delivery. At the same time, all unused products were retrieved for inventory. Product compliance was acquired by counting the number of the product's doses returned at the time of specified clinic visits.

Statistical analysis. Data were analyzed by intention to treat using SPSS version 25.0 for Windows. We compared characteristics and changes in questionnaire scores between hospitalized and non-hospitalized patients. Normally distributed baseline characteristics of the population were compared using unpaired Student's $t$ test, non-normally distributed ones by Mann-Whitney- $U$ test. Questionnaire scores were compared by Wilcoxon rank test. All data are shown as means and SD. A $p$ level of $<0.05$ was considered significant for all tests.

Results. At the baseline, the two patient groups were comparable in main clinical and laboratory parameters (Table I). Moreover, all participants were treated with ACE inhibitors/sartans and statins.

Table I. Main clinical and laboratory parameters of the enrolled patients

\begin{tabular}{|c|c|c|c|c|c|}
\hline Parameter & $\begin{array}{l}\text { Hospitalized } \\
\qquad(N=19)\end{array}$ & $\begin{array}{l}\text { Home-cared } \\
(N=21)\end{array}$ & Parameter & $\begin{array}{l}\text { Hospitalized } \\
(N=19)\end{array}$ & $\begin{array}{l}\text { Home-cared } \\
\qquad(N=21)\end{array}$ \\
\hline Age [years] & $68.9 \pm 3.3$ & $68.2 \pm 3.5$ & $\begin{array}{l}\text { Total cholesterol } \\
{[\mathrm{mg} / \mathrm{dl}]}\end{array}$ & $237.8 \pm 21.7$ & $238.3 \pm 23.0$ \\
\hline $\begin{array}{l}\text { Waist circumference } \\
{[\mathrm{cm}]}\end{array}$ & $90.7 \pm 4.5$ & $88.3 \pm 7.1$ & $\begin{array}{l}\text { HDL cholesterol } \\
{[\mathrm{mg} / \mathrm{dl}]}\end{array}$ & $46.5 \pm 4.5$ & $44.1 \pm 3.7$ \\
\hline $\begin{array}{l}\text { Body mass index } \\
{\left[\mathrm{kg} / \mathrm{m}^{2}\right]}\end{array}$ & $23.7 \pm 1.7$ & $22.8 \pm 2.2$ & $\begin{array}{l}\text { LDL cholesterol } \\
{[\mathrm{mg} / \mathrm{dl}]}\end{array}$ & $144.7 \pm 17.9$ & $151.4 \pm 16.5$ \\
\hline $\begin{array}{l}\text { Systolic blood } \\
\text { pressure [mm Hg] }\end{array}$ & $137.3 \pm 4.8$ & $136.5 \pm 5.6$ & $\begin{array}{c}\text { Non-HDL } \\
\text { cholesterol [mg/dl] }\end{array}$ & $191.4 \pm 20.7$ & $194.7 \pm 21.4$ \\
\hline $\begin{array}{l}\text { Diastolic blood } \\
\text { pressure [mm Hg] }\end{array}$ & $88.7 \pm 3.2$ & $87.6 \pm 5.2$ & $\begin{array}{l}\text { Triglycerides } \\
{[\mathrm{mg} / \mathrm{dl}]}\end{array}$ & $235.4 \pm 23.7$ & $216.8 \pm 16.1$ \\
\hline $\begin{array}{l}\text { Serum uric acid } \\
{[\mathrm{mg} / \mathrm{dl}]}\end{array}$ & $5.3 \pm 0.9$ & $5.4 \pm 0.8$ & gGT [mg/dl] & $34.3 \pm 4.4$ & $32.9 \pm 4.5$ \\
\hline $\begin{array}{l}\text { Serum creatinine } \\
{[\mathrm{mg} / \mathrm{dl}]}\end{array}$ & $0.7 \pm 0.3$ & $0.7 \pm 0.4$ & $\mathrm{CPK}[\mathrm{U} / \mathrm{ml}]$ & $141.3 \pm 31.3$ & $154.7 \pm 29.2$ \\
\hline
\end{tabular}


Table II. Modification of the biometric test carried out on the volunteers in both treatment groups

\begin{tabular}{|lcccc|}
\hline Parameter & \multicolumn{2}{c|}{ Hospitalized $(N=19)$} & \multicolumn{2}{c|}{ Home-cared $(N=21)$} \\
\cline { 2 - 5 } & Pre-treatment & Post-treatment & Pre-treatment & Post-treatment \\
\hline MMSE & $24.1 \pm 1.0$ & $24.9 \pm 0.7^{*}$ & $23.9 \pm 0.9$ & $25.0 \pm 0.9^{*}$ \\
\hline PSQ Index & $2.8 \pm 0.5$ & $2.1 \pm 0.6^{*}$ & $2.9 \pm 0.6$ & $2.3 \pm 0.7^{*}$ \\
\hline SRDS & $43.9 \pm 5.5$ & $36.2 \pm 4.9^{*}$ & $44.5 \pm 5.3$ & $37.8 \pm 5.7^{*}$ \\
\hline PCFS Scale & $3.2 \pm 0.4$ & $2.8 \pm 0.4^{*}$ & $3.1 \pm 0.3$ & $2.7 \pm 0.2^{*}$ \\
\hline
\end{tabular}

MMSE - Mini-Mental State Examination, PSQ - Perceived Stress Questionnaire, SRDS - Self-Rating Depression Scale, PCFS - PostCOVID-19 Functional Status. ${ }^{*} P<0.05$ vs. baseline.

Each enrolled subject completed the study. Two patients conveyed an unpleasant aftertaste after nutraceutical intake. Compliance to products was similar between patient groups: $96 \%$ in hospitalized patients, $92 \%$ in home-cared ones $(p>0.05)$.

On a scale from 1 to 10 , the mean acceptability was 9 for both groups.

The main results of the trial are reported in Table II. MMSE, PSQ Index, SRDS and PCFS scale significantly improved in both patient groups.

Discussion. The main limitations of the study are the small sample size and the relatively shortterm observation. Therefore, the observed results should be confirmed in larger and long-term studies. In particular, since Synaid was never tested in this specific kind of patients it was not possible to estimate an adequate sample size. Anyway, the improvements observed are in line with those measured in our previous investigation on patients with known cognitive decline [6]. Moreover, the effects were also those expected considering the antioxidant, cholinesterase inhibition and serotonin-reuptake inhibition effects of the Synaid components [6, 11]. Then, we applied a limited number of tests. Further investigations may indicate that cognitive, anxiety or depression components are more significantly improved by the tested product. However, the improvement of the PCFS scale could be particularly interesting due to the increased worrying about the long-term consequences of SARS-CoV-2 infection. Finally, we did not measure the level of proinflammatory cytokines, which could have contributed to the postCOVID memory impairment [12]. However, we selected patients recovered from COVID with mild neurological symptoms where probably the residual systemic inflammation was not so relevant.

In conclusion, the tested combined nutraceutical can significantly improve functional status, MMSE and the main psychological disorders associated with mild cognitive improvement versus baseline after Sars-Cov-2 infection, both in hospitalized patients and in home-cared patients, without side effects.

\section{Conflict of interest}

The authors declare no conflict of interest.

\section{References}

1. Mancusi C, Grassi G, Borghi C, et al.; SARS-RAS Investigator Group. Clinical characteristics and outcomes of patients with COVID-19 infection: the results of the SARS-RAS study of the Italian Society of Hypertension. High Blood Press Cardiovasc Prev 2021; 28: 5-11.

2. Zhou H, Lu S, Chen J, et al. The landscape of cognitive function in recovered COVID-19 patients. J Psychiatr Res 2020; 129: 98-102.

3. Heneka MT, Golenbock D, Latz E, Morgan D, Brown R. Immediate and long-term consequences of COVID-19 infections for the development of neurological disease. Alzheimers Res Ther 2020; 12: 69.

4. Ritchie K, Chan D, Watermeyer T. The cognitive consequences of the COVID-19 epidemic: collateral damage? Brain Commun 2020; 22: fcaa069.

5. Ferrari R, Maggioni AP, Tavazzi L, Rapezzi C. The battle against COVID-19: mortality in Italy. Eur Heart J 2020; 41: 2050-2.

6. Cicero AF, Bove M, Colletti A, et al. Short-term impact of a combined nutraceutical on cognitive function, perceived stress and depression in young elderly with cognitive impairment: a pilot, double-blind, randomized clinical trial. J Prev Alzh Dis 2017; 4: 12-5.

7. Crum RM, Anthony JC, Bassett SS, Folstein MF. Population-based norms for the mini-mental state examination by age and educational level. JAMA 1993; 269: 2386-91.

8. Fliege $\mathrm{H}$, Rose $M$, Arck P, et al. The Perceived Stress Questionnaire (PSQ) reconsidered: validation and reference values from different clinical and healthy adult samples. Psychosom Med 2005; 67: 78-88.

9. Zung WW. A self-rating depression scale. Arch Gen Psych 1965; 12: 63-70.

10. Klok FA, Boon GJAM, Barco S, et al. The post-COVID-19 Functional Status scale: a tool to measure functional status over time after COVID-19. Eur Respir J 2020; 56 : 2001494.

11. Cicero AFG, Fogacci F, Banach M. Botanicals and phytochemicals active on cognitive decline: the clinical evidence. Pharmacol Res 2018; 130: 204-12.

12. Cothran TP, Kellman S, Singh S, et al. A brewing storm: the neuropsychological sequelae of hyperinflammation due to COVID-19. Brain Behav Immun 2020; 88: 957-8. 